\title{
Hepatic arterial infusion enhances DOTATOC radiopeptide therapy in patients with neuroendocrine liver metastases
}

\author{
Clemens Kratochwil, Ruben López-Benítez ${ }^{1}$, Walter Mier, Sabine Haufe, \\ Berend Isermann ${ }^{2}$, Hans-Ulrich Kauczor ${ }^{1}$, Peter L Choyke ${ }^{3}$, Uwe Haberkorn \\ and Frederik L Giesel
}

Department of Nuclear Medicine, University Hospital Heidelberg, INF 400, 69120 Heidelberg, Germany

Departments of ${ }^{1}$ Diagnostic and Interventional Radiology and ${ }^{2}$ Endocrinology, University Hospital Heidelberg, Heidelberg, Germany

${ }^{3}$ Molecular Imaging Program, National Cancer Institute, Bethesda, Maryland, USA

(Correspondence should be addressed to C Kratochwil; Email: clemens.kratochwil@ med.uni-heidelberg.de)

\begin{abstract}
Intravenously administered radiolabeled peptides targeting somatostatin receptors are used for the treatment of unresectable gastroenteropancreatic neuroendocrine tumors (GEP-NETs). Recently, we demonstrated a high first-pass effect during intra-arterial (i.a.) administration of positron emission tomography (PET) labeled ${ }^{68} \mathrm{Ga}$-DOTA ${ }^{0}$-D-Phe ${ }^{1}-\mathrm{Tyr}^{3}$-octreotide (DOTATOC). In this pilot study, we investigated the therapeutic effectiveness of arterial administered DOTATOC, labeled with the therapeutic $\beta$ emitters ${ }^{90} \mathrm{Y}$ and ${ }^{177} \mathrm{Lu} .{ }^{90} \mathrm{Y}$ - and/or ${ }^{177} \mathrm{Lu}$-DOTATOC were infused into the hepatic artery of 15 patients with liver metastases arising from GEP-NETs. Response was assessed using DOTATOC-PET, multiphase contrast enhanced computed tomography, magnetic resonance imaging, and the serum tumor marker chromogranin A. Pharmacokinetic data of the arterial approach were assessed using ${ }^{111}$ In-DOTATOC scans. With the treatment regime of this pilot study, complete remission was achieved in one (7\%) patient and partial remission was observed in eight $(53 \%)$ patients, six patients were classified as stable $(40 \%$; response evaluation criteria in solid tumors criteria). The concomitant decrease of elevated serum tumor marker confirmed the radiologic response. Median time to progression was not reached within a mean follow-up period of 20 months. Receptor saturation and redistribution effects were identified as limiting factors for i.a. DOTATOC therapy. The high rate of objective radiologic response in NET patients treated with arterial infusion of ${ }^{90} \mathrm{Y}-\mathrm{I}^{177} \mathrm{Lu}$-DOTATOC compares favorably with systemic chemotherapy and intravenous radiopeptide therapy. While i.a. DOTATOC therapy is only applicable to patients with tumors of limited anatomic distribution, the results of this pilot study are a promising development in the treatment of GEP-NET and warrants further investigation of this novel approach.
\end{abstract}

Endocrine-Related Cancer (2011) 18 595-602

\section{Introduction}

Radiopeptides targeting somatostatin receptors, such as ${ }^{90} \mathrm{Y}$ - or ${ }^{177}$ Lu-labeled DOTA ${ }^{0}$-D-Phe ${ }^{1}-\mathrm{Tyr}^{3}$-octreotide (DOTATOC) or DOTA ${ }^{0}$-D-Phe ${ }^{1}-\mathrm{Tyr}^{3}$-octreotate (DOTATATE), have been under investigation as a treatment for metastatic neuroendocrine tumors (NETs) for over 10 years. Clinical studies have demonstrated objective radiologic responses in up to $30 \%$ of the patients (Kwekkeboom et al. 2008, Bodei et al. 2010). Patients with gastroenteropancreatic (GEP)-NETs frequently develop hepatic metastases that are often the life-limiting factor. The tendency for GEP-NET to metastasize to the liver and seldom to other organs suggests that intra-arterial (i.a.) therapies might be superior to systemic treatments. Using ${ }^{68} \mathrm{Ga}$-DOTATOC, a positron emission tomography (PET) emitting radiopeptide targeting somatostatin receptors, it was shown that i.a. administration led to a 3.7-fold higher tumor accumulation compared with i.v. infusion (Kratochwil et al. 2010) in the early-uptake phase. However, due to the short half-life of ${ }^{68} \mathrm{Ga}\left(t_{1 / 2}=68 \mathrm{~min}\right)$ only the initial tumor concentrations could be assessed. It still remained 
unclear if redistribution effects would decrease the observed improvement over time and therefore, if the i.a. approach would translate into better therapeutic outcomes. In this pilot study, we evaluate the efficacy of i.a. ${ }^{90} \mathrm{Y}$ - and ${ }^{177} \mathrm{Lu}$-DOTATOC therapy in liver metastatic GEP-NET. In addition, the pharmacokinetics of i.a. DOTATOC is further investigated by the diagnostic surrogate ${ }^{111}$ In-DOTATOC $\left(t_{1 / 2}=67 \mathrm{~h}\right)$.

\section{Materials and methods}

\section{Patients}

The study included patients with histologically confirmed, multifocal liver metastases arising from well-differentiated $(\mathrm{G} 1 / \mathrm{G} 2)$ progressive GEP-NET. These patients were not surgical candidates. Patients with extraabdominal disease were excluded. Fifteen patients (six men, nine women, and age 40-65 years) were entered the study including patients with gastric NET $(n=2)$, enteric NET $(n=4)$, pancreatic NET $(n=8)$, and one patient with an unknown primary (Table 1). A positive tumor somatostatin receptor expression was validated with ${ }^{68}$ Ga-DOTATOC PET/computed tomography (CT). The therapies were conducted in accordance with the Helsinki Declaration and our national regulations; written informed consent was obtained from all patients.

\section{Diagnostic imaging}

Staging and restaging procedures included contrast enhanced CT and ${ }^{68}$ Ga-DOTATOC PET/CT. Contrast

Table 1 Overview of the patient cohort, follow-up, and tumor markers

\begin{tabular}{|c|c|c|c|c|c|c|c|c|}
\hline Patient & Gender & $\begin{array}{l}\text { Primary } \\
\text { tumor }\end{array}$ & $\begin{array}{c}\text { Cycles/activity } \\
\text { (GBq) }\end{array}$ & $\begin{array}{c}\mathbf{C h r A}_{\text {initial }} \\
(\mathrm{ng} / \mathrm{ml})\end{array}$ & $\begin{array}{l}\text { ChrA } \mathbf{A}_{\text {post }} \\
(\mathrm{ng} / \mathrm{ml})\end{array}$ & $\begin{array}{l}\text { Change } \\
\text { ChrA (\%) }\end{array}$ & RECIST & Course of follow-up \\
\hline 1 & M & G-NET & $4 / 4 Y+18 \mathrm{Lu}$ & 10 & n.e.a.b. & & -100 & CR $>27$ months; last ChrA 6.3 \\
\hline 2 & $\mathrm{~F}$ & P-NET & 3/12 Y + $4 \mathrm{Lu}$ & 50 & 23.1 & -53.8 & -57 & $\begin{array}{l}\text { SD within partial remission }>13 \\
\text { months; last ChrA } 17.6\end{array}$ \\
\hline 3 & $\mathrm{~F}$ & P-NET & $3 / 12.5 \mathrm{Y}+4 \mathrm{Lu}$ & 3069 & 302 & -90.16 & -50.69 & $\begin{array}{l}\text { PD (bone) after } 12 \text { months } \\
\text { (ChrA 202.8); SAx; PD (liver) } \\
\text { after } 18 \text { months; last ChrA } \\
\text { 4158; CTx }\end{array}$ \\
\hline 4 & $\mathrm{~F}$ & P-NET & $2 / 8 Y+8 L u$ & 120 & 48.2 & -59.83 & -50 & $\begin{array}{l}\text { SD within partial remission }>13 \\
\text { months; last ChrA } 97.4\end{array}$ \\
\hline 5 & M & P-NET & $3 / 12 \mathrm{Y}+12 \mathrm{Lu}$ & 33 & 15.9 & -51.82 & -48.56 & $\begin{array}{l}\text { SD within partial remission }>25 \\
\text { months; last ChrA } 18.5\end{array}$ \\
\hline 6 & $\mathrm{~F}$ & P-NET & $4 / 22 \mathrm{Y}+12 \mathrm{Lu}$ & 131 & 24.5 & -81.3 & -43.98 & $\begin{array}{l}\text { Further PR for } 23 \text { months; } \\
\text { re-evaluation: downstaged to } \\
\text { surgery; last ChrA }<5\end{array}$ \\
\hline 7 & $\mathrm{~F}$ & P-NET & $5 / 18 \mathrm{Y}+14 \mathrm{Lu}$ & 133.9 & 13.3 & -90.07 & -42.81 & $\begin{array}{l}\text { SD within partial remission }>25 \\
\text { months; last ChrA } 17.9\end{array}$ \\
\hline 8 & $\mathrm{~F}$ & P-NET & $4 / 16 \mathrm{Y}+16 \mathrm{Lu}$ & 41.5 & 38.2 & -7.95 & -40.07 & $\begin{array}{l}\text { SD within partial remission }>26 \\
\text { months; last ChrA } 41.7\end{array}$ \\
\hline 9 & $\mathrm{~F}$ & P-NET & $3 / 4 Y+24 L u$ & 86.8 & 32.1 & -63.02 & -35 & $\begin{array}{l}\text { SD within partial remission }>22 \\
\text { months under SAx; last ChrA } \\
64.7\end{array}$ \\
\hline 10 & M & CUP-NET & $2 / 14 \mathrm{Y}+2 \mathrm{Lu}$ & 171.5 & 64.5 & -62.39 & -24.29 & SD $>15$ months last ChrA 50.2 \\
\hline 11 & $\mathrm{~F}$ & Jejunum & $2 / 8 \mathrm{Y}+8 \mathrm{Lu}$ & 45.4 & 45.7 & 0.66 & -5.33 & $\begin{array}{l}\text { SD > 18; SIRT due to recurrent } \\
\text { diarrhea; SD > } 5 \text { months; last } \\
\text { ChrA 25.1 }\end{array}$ \\
\hline 12 & M & Jejunum & $2 / 6 \mathrm{Y}+6.4 \mathrm{Lu}$ & 330 & 385 & 16.67 & -4.55 & SD > 19; last ChrA 221.5 \\
\hline 13 & M & Jejunum & $2 / 6 \mathrm{Y}+4 \mathrm{Lu}$ & 1100 & 429 & -61 & 0 & SD > 13; last ChrA 130.8; SAx \\
\hline 14 & $\mathrm{~F}$ & G-NET & $4 / 16 \mathrm{Y}+13 \mathrm{Lu}$ & 902 & 1462 & 62.08 & 3.43 & $\begin{array}{l}\text { PD (bone) after } 11 \text { months; } \\
\text { ChrA 2651; CTx; death after } \\
5 \text { months }\end{array}$ \\
\hline 15 & M & Illeocoecal & $3 / 12 \mathrm{Y}+10.4 \mathrm{Lu}$ & 95.9 & 102 & 6.36 & 7.7 & $\begin{array}{l}\text { PD (liver) after } 12 \text { months; } 3 \\
\text { additional cycles i.a. DOTA- } \\
\text { TOC; PD (liver) after }>6 \\
\text { months; last ChrA } 370.7\end{array}$ \\
\hline
\end{tabular}

Cycles, number of treatments; activity, cumulative prescribed activity; Y, Yttrium-90; Lu, Lutetium-177; ChrA, chromogranin A; $\mathrm{M}$, male; F, female; P-NET, pancreatic neuroendocrine tumor; G-NET, gastric neuroendocrine tumor; CUP-NET, neuroendocrine tumor of unknown primary; PD, progressive disease; SD, stable disease; SAx, treatment with somatostatin analogues; CTx, chemotherapy; SIRT, radioembolization; n.e.a.b., not elevated at baseline. 
enhanced magnetic resonance imaging (MRI) using a hepatocyte-specific contrast agent was also performed.

A diagnostic CT was performed as part of the PET/CT examination (Biograph 6, Siemens/CTI, Knoxville, TN, USA). Contrast media (Imeron 400, Bracco Diagnostics, Inc., Princeton, NJ, USA) was injected at a flow rate of $4 \mathrm{cc} / \mathrm{s}$. The liver and abdomen were initially examined without contrast media (130 keV, 30 mAs, CareDose, Siemens/CTI), followed by an arterial phase ( $25 \mathrm{~s}$ delay) and a portal venous phase (60 s delay). Slice collimation was $6 \times 2.0 \mathrm{~mm}$, pitch factor $1.5,110 \mathrm{kV}, 95 \mathrm{mAs}$ (CareDose), matrix $512 \times 512$, rotation time $0.6 \mathrm{~s}$, and secondary raw data were reformatted to a slice thickness of $5 \mathrm{~mm}$ with a $2.5 \mathrm{~mm}$ increment.

PET studies were also performed on the PET/CT (Siemens/CTI). Imaging was initiated $45 \pm 5$ min after i.v. injection of $84-196 \mathrm{MBq}{ }^{68} \mathrm{Ga}$-DOTATOC. The synthesis of the tracer has already been described previously (Kratochwil et al. 2010). Static emission scans, corrected for dead time, scatter, and decay were acquired from the vertex to the proximal legs requiring eight bed positions, 4 min each. $\mathrm{CT}$ without contrast agent was used for attenuation correction. The images were iteratively reconstructed with the ordered subset expectation maximisation (OSEM) algorithm using four iterations with eight subsets and Gaussian filtering to achieve an in-plane spatial resolution of $5 \mathrm{~mm}$ at full-width half-maximum.

MRI was performed on a $1.5 \mathrm{~T}$ whole body system (Avanto 1.5 T, Siemens Medical Solutions, Erlangen, Germany) using Gd-EOB-DTPA, a hepatocytespecific contrast agent (Primovist, Bayer-Schering). Contrast enhanced MRI was performed by a volumetric interpolated breath-hold examination (VIBE) $(\mathrm{TR}=3.32 \mathrm{~ms}, \mathrm{TE}=1.08 \mathrm{~ms}$, matrix: $320 \times 180)$; hepatocyte phase was acquired $10 \mathrm{~min}$ after injection. Standard fat saturated $\mathrm{T} 1 \mathrm{w}$ and $\mathrm{T} 2 \mathrm{w}$ were also performed.

Radiologic response was determined using response evaluation criteria in solid tumors (RECIST 1.0) guidelines for tumor size. The sum of the diameters of up to five liver lesions was compared with a followup examination 10-12 weeks after the last cycle of therapy. PET/CT and MRI examinations were repeated every 3 months during the follow-up period.

\section{Toxicity assessment}

Common Terminology Criteria for Adverse Events v3.0 as published by the National Cancer Institute (Bethesda, MD, USA) are used to score side effects during therapy. Acute hematologic and chronic renal toxicity are known to be most important for systemic peptide receptor radiation therapy (PRRT). To assess hepatic toxicity, liver enzymes (e.g. $\gamma \mathrm{GT}$, ALT, and AST) were obtained. Furthermore, before each therapeutic PRRT intervention, as well as every 3 months after the last treatment, a nuclear renal scan $\left({ }^{99 m}\right.$ Tc-MAG3) was performed and plasma clearance was calculated according to the Bubeck formula (E.Soft, Siemens).

\section{Arterial catheter}

A 4-French catheter (Sidewinder-S1) was placed in the celiac artery via a transfemoral approach using the Seldinger technique under local anesthesia. Then a microcatheter (Progreat, Terumo, Tokyo, Japan) was inserted coaxially and advanced into the common hepatic artery or proper hepatic artery as required by the location of the metastases. In cases of anatomic variation, the most appropriate arterial feeder was catheterized. Catheter and sheath were heparinized (1500 units) and fixed for the i.a. DOTATOC application.

\section{Synthesis of the radiopeptides}

DOTATOC (molecular weight $1421 \mathrm{~g} / \mathrm{mol}$ ) was synthesized as described previously (Kratochwil et al. 2010). Our kit formulation contains $100 \mu \mathrm{g}$ DOTATOC acetate, $7.5 \mathrm{mg}$ gentisic acid (Fluka, Basel, Switzerland), and $0.4 \mathrm{M}$ sodium acetate buffer in $400 \mu \mathrm{l}$ water at $\mathrm{pH} 5$ (Sigma-Aldrich). Either one kit is mixed with $4 \mathrm{GBq}\left[{ }^{177} \mathrm{Lu}\right]$ lutetium chloride (Perkin Elmer, Rodgau, Germany) or the same kit containing $50 \mu \mathrm{g}$ DOTATOC acetate is mixed with $4 \mathrm{GBq}\left[{ }^{90} \mathrm{Y}\right] \mathrm{yttrium}$ chloride (Eckert \& Ziegler, Berlin, Germany). The solution is kept at $95{ }^{\circ} \mathrm{C}$ for $30 \mathrm{~min}$. After cooling $2 \mathrm{ml}$ of $0.9 \%$ sodium chloride is added and the product is sterile filtered. The specific activity is $40 \mathrm{MBq}{ }^{177} \mathrm{Lu}$ or $80 \mathrm{MBq}{ }^{90} \mathrm{Y}$ per microgram peptide.

\section{Therapeutic regimes}

The type of radionuclide was selected depending on the tumor configuration. If the main tumor burden (based on the enhanced MRI) was localized in lesions with a diameter of $>2 \mathrm{~cm},{ }^{90} \mathrm{Y}$ (maximum tissue range: $11 \mathrm{~mm}$ ) was selected, but if the lesions were $<2 \mathrm{~cm}$ then ${ }^{177} \mathrm{Lu}$ (maximum tissue range: $3 \mathrm{~mm}$ ) was selected. If the patient had a heterogeneous size distribution then a combination of both radionuclides was prescribed. The rationale for this concept has been described previously (de Jong et al. 2005). The therapeutic activity was correlated with the tumor 
volume (assessed with MRI) and ranged from $2 \mathrm{GBq}$ ${ }^{177} \mathrm{Lu}$ (in a patient with $<1 \mathrm{ml}$ tumor tissue) to $8 \mathrm{GBq}$ ${ }^{90} \mathrm{Y}$ (in a patient with $>2 \mathrm{ml}$ tumor tissue). Infusion speed was $50 \mu \mathrm{g}$ peptide/h. A lysine $30 \mathrm{~g} / \mathrm{l}$ and arginine $30 \mathrm{~g} / \mathrm{l}$ solution was co-infused at $200 \mathrm{ml} / \mathrm{h}$ for nephroprotection (Rolleman et al. 2003). Concomitant medication also included Ondansetron (Kevatril) and sufficient hydration was administered.

\section{Imaging of pharmacokinetics}

In four patients, we intra-individually compared the time-activity-curves after both i.v. and i.a. infusion of a typical therapeutic amount of DOTATOC peptide $(150 \mu \mathrm{g})$ labeled with ${ }^{111}$ In-DOTATOC (Indium-111; $\gamma$ emitter, and half-life 2.8 days). First, we performed $40 \mathrm{~min}$ of dynamic imaging following either the i.v. or i.a. infusion (infusion time: $20 \mathrm{~min}$ ) of $250 \mathrm{MBq}$ ${ }^{111}$ In-DOTATOC. Then, planar whole body scans were acquired after $1,4,24,48$, and $72 \mathrm{~h}$ and single photon emission CT (SPECT)/CT (Hawekeye, GE Healthcare, Little Chalfont, Bucks, UK) imaging of the abdomen was performed after $24 \mathrm{~h}$ respectively. Scatter and attenuation correction were performed in planar imaging (triple energy window, geometric mean) and SPECT/CT imaging. Regions of interest (ROIs) were drawn around targeted and non-targeted lesions and time-activity-curves were created.

\section{Results}

\section{Treatment response}

According to RECIST criteria, we found one (7\%) complete response (CR) and eight partial remissions (53\%). Six patients $(40 \%)$ were classified as stable disease (SD). No patient had progressive disease under therapy. Within a mean follow-up period of 20 months remissions and SDs sustained in most patients. Only three patients relapsed and median time to progression (TTP) is still not reached. Figure 1 demonstrates the example of CR, which is now persisting for more than 27 months. Figure 2 illustrates a typical patient with partial remission (PR). The radiologic response (RECIST criteria), as assessed 3 months after the final PRRT, is summarized in Fig. 3. The results of the follow-up examinations (PET/CT and MRI every 3 months) are given in the last column of Table 1.

In 14 of the 15 patients, an elevated serum chromogranin A (CgA) was present before treatment. In 10 of the 14 patients, the tumor marker was more than threefold the upper limit of normal $(25 \mathrm{U} / \mathrm{ml}$; DAKO, Glostrup, Denmark). After, 3 months, the last cycle the elevated $\mathrm{CgA}$ declined by a mean of $53 \%$. One patient (no. 14) was developed bone marrow infiltration and $\mathrm{CgA}$ increased appreciably; however, the hepatic metastases remained stable (Table 1).

\section{Toxicity and liver function}

None of the 15 patients experienced acute side effects or unexpected complications. Known side effects of PRRT include acute hematologic and chronic renal toxicity. Nausea and emesis are associated with the nephroprotective solution.

All patients had normal creatinine and urea as well as a normal MAG3 clearance. After 3 months, the last cycle there was no significant change in these parameters.

One patient developed grade III thrombocytopenia, leukopenia, and decreased hemoglobin with prolonged recovery; grade I toxicity was still present 6 months after treatment. In all other patients only grades I and II thrombocytopenia was present and recovered to normal within 3 months in 12 patients and within 6 months in two patients. (a)

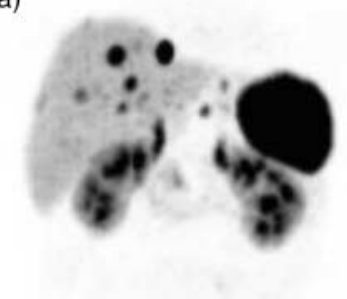

(b)

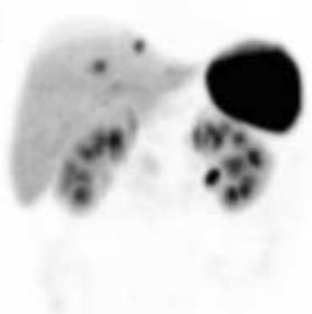

(c)

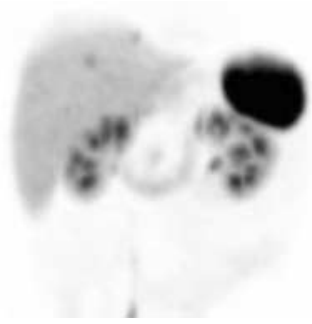

(d)

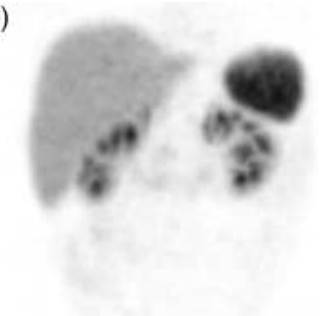

Figure 1 Complete remission. Maximum intensity projections of initial and follow-up examinations with ${ }^{68}$ Ga-DOTATOC (DOTA ${ }^{0}$-D$\mathrm{Phe}^{1}-\mathrm{Tyr}^{3}$-octreotide) positron emission tomography (PET). A patient presented with 12 neuroendocrine liver metastases as diagnosed with magnetic resonance imaging (MRI) and ${ }^{68} \mathrm{Ga}-\mathrm{DOTATOC}-\mathrm{PET}$. (a) After each cycle of intra-arterial radiopeptide therapy regression of the metastases was observed ( $b$ and $c)$. After three cycles complete remission was demonstrated both in MRI and DOTATOC-PET (d). A fourth cycle was performed for consolidation and the complete remission is still present with 27 months of follow-up. 

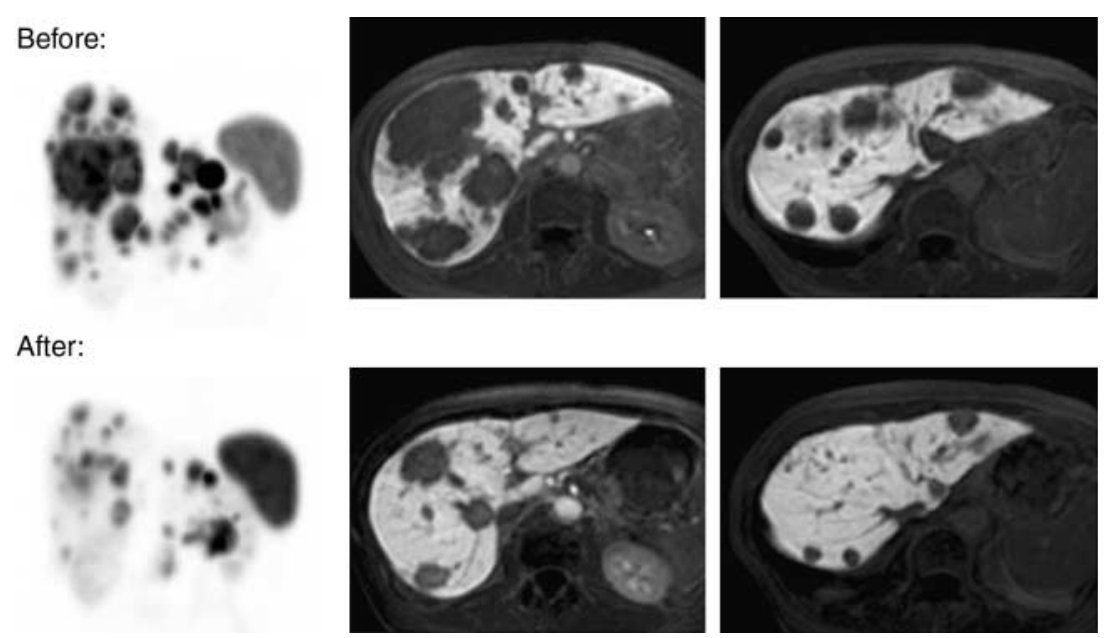

Figure 2 Partial remission. Patient with multiple neuroendocrine liver metastases before (upper row) and after (lower row) one cycle of intra-arterial therapy with $4 \mathrm{GBq}{ }^{90} \mathrm{Y}$ - and $4 \mathrm{GBq}{ }^{177} \mathrm{Lu}$-labeled DOTATOC. DOTATOC-PET is presented as maximum intensity projections (left) and with contrast enhanced MRI follow-up studies. Serum chromogranin A decreased by $90 \%$.

Bilirubin, cholinesterase, and prothrombin ratio were not significantly affected by treatment. The course of $\gamma$ GT was different between the responders (CR and PR) and non-responders (SD). While in the non-responder group the mean $\gamma \mathrm{GT}$ increased from baseline (97.5 U/l) to follow-up (159.5 U/l), the value improved in the responder group from 69.4 to $33.6 \mathrm{U} / \mathrm{l}$. The transaminases showed a comparable course to that of $\gamma \mathrm{GT}$.

\section{Pharmacokinetics}

During i.v. infusion of ${ }^{111}$ In-DOTATOC peptide the count rate within the tumor ROI increased almost linearly. After stopping the infusion the tumor uptake continued to increase until a maximum was reached at $4 \mathrm{~h}$ post-injection. In contrast, during i.a. infusion (same protocol) the slope of the time-activity-curve (TAC) decreased over time. The maximum tumor uptake was observed just after finishing the infusion with a count rate 3.5-fold higher compared with the i.v. value. Adjunct, there was a relevant washout of the radiopeptide from the target lesion and the improvement of tumor uptake after i.a. infusion decreased to a twofold ratio until $4 \mathrm{~h}$ post-injection. After that, the uptake ratio was more stable and only a slow decrease to 1.3 -fold was observed until $72 \mathrm{~h}$ post-injection (Fig. 4).

\section{Discussion}

Metastatic GEP-NETs often present a therapeutic challenge. The majority of tumors are diagnosed at a time when the only curative treatment, radical surgery, is no longer an option. Hepatic metastases are common and often are eventually lethal. Depending on size, location, and number, some of them may be amenable to surgical resection or radiofrequency ablation. If this is not feasible, transarterial embolization (TAE), transarterial chemoembolization (TACE), or selective radioembolization (SIRT) can be used (Modlin et al. 2006). However, these non-targeted therapies also harm normal liver parenchyma and, therefore, are associated with off-target side effects (e.g. the postembolization syndrome). Conventional chemotherapies are only moderately effective in

Treatment induced changes in sum diameter of five liver metastases

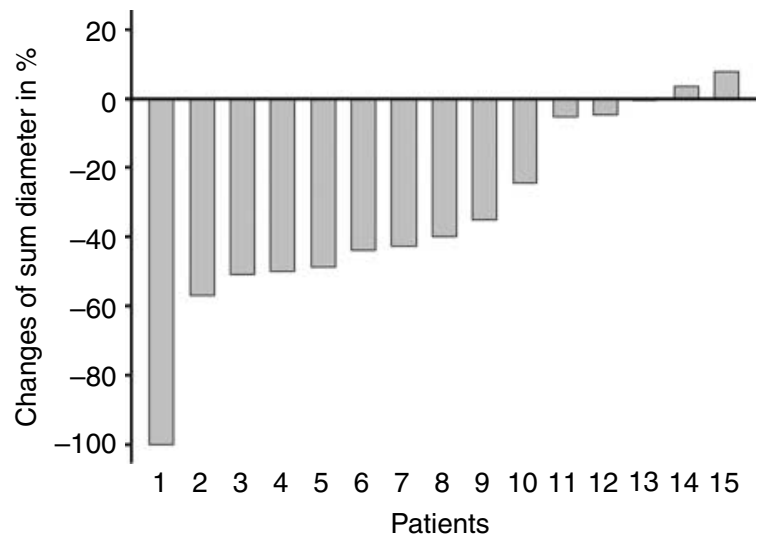

Figure 3 Waterfall graph demonstrating the radiologic response. Treatment induced changes in sum of the diameter of up to five liver metastases according to Response Evaluation Criteria In Solid Tumors (RECIST) 1.0 criteria of 15 patients (patient numbering corresponding to Table 1). Overall response (complete and partial remission) was observed in nine of the 15 patients $(60 \%)$. 


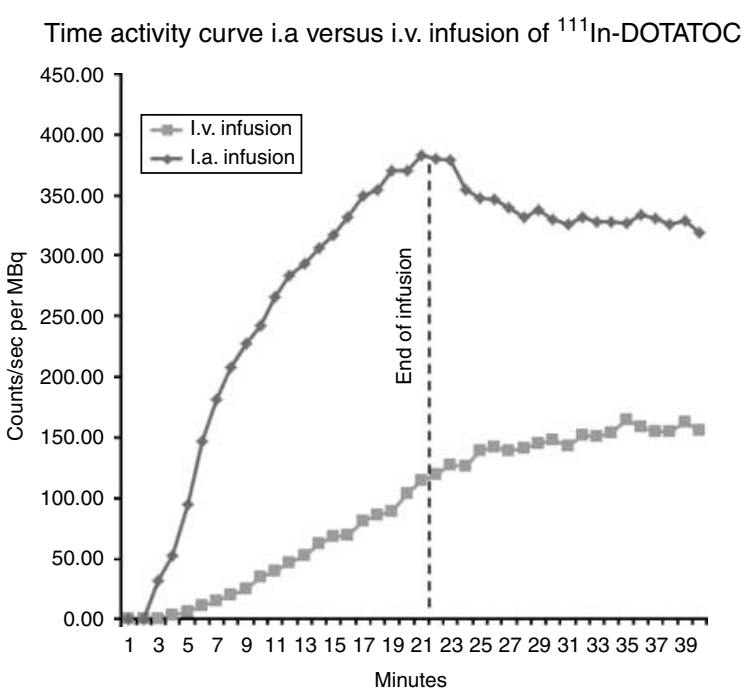

Figure 4 Pharmacokinetics. Tumoral time-activity-curves for 20 min intra-arterial versus i.v. infusion of ${ }^{111}$ In-DOTATOC. Arterial infusion leads to a higher slope in the initial phase, but after $10 \mathrm{~min}$ the slope decreases due to saturating effects and is followed by a washout phase. In contrast, the i.v. infusion uptake demonstrates a nearly linear slope and continues to increase after the infusion is stopped.

pancreatic GEP-NETs and poorly effective in midgut GEP-NETs (Strosberg et al. 2011). Long-term therapy with somatostatin analogs is focused on symptom amelioration and in the improvement in quality of life. In addition, some antiproliferative effects were observed but these effects are cytostatic rather than cytotoxic (Rinke et al. 2009). Systemic (i.v.) peptide receptor targeted radiotherapy using radiolabeled somatostatin analogs was introduced one decade ago. With this well-tolerated treatment, objective radiologic response can be observed in up to $30 \%$ of the patients, but in most circumstances it still has only a tumor stabilizing effect (Kwekkeboom et al. 2008, Bodei et al. 2010). Recently, we demonstrated a strong first-pass effect of i.a. administered ${ }^{68} \mathrm{Ga}$-DOTATOC using quantitative PET-imaging and we hypothesized that this observation might be transferable to the therapeutic radioisotopes such as $\beta$-particle emitting ${ }^{90} \mathrm{Y}$ - and ${ }^{177} \mathrm{Lu}$-DOTATOC (Kratochwil et al. 2010). However, the imaging agent ${ }^{68} \mathrm{Ga}$-DOTATOC has a very short physical half-life $\left({ }^{68} \mathrm{Ga} t_{1 / 2}=1.13 \mathrm{~h}\right)$ and it was unclear whether the increase of early receptor binding would predict the therapeutic efficacy of the ${ }^{90} \mathrm{Y}$ - or ${ }^{177} \mathrm{Lu}$-labeled peptide which is also determined by the biological half-life (including washout and redistribution effects) of the peptide and the physical half-life of the radiometal $\left({ }^{90} \mathrm{Y} t_{1 / 2}=64 \mathrm{~h}\right.$ and ${ }^{177} \mathrm{Lu}$ $t_{1 / 2}=160 \mathrm{~h}$ ). In addition, the much higher dose of peptide given during therapy could affect kinetics compared with the trace doses used for imaging (Velikyan et al. 2010). Therefore, we compared the time-activity-curves after i.v. and i.a. infusion of a typical therapeutic dose of peptide which was labeled with a long half-life $\gamma$ emitting radionuclide $\left({ }^{111} \mathrm{In}\right.$ $t_{1 / 2}=67 \mathrm{~h}$ ). In contrast to i.v. infusion, we observed a saturating curve followed by an initial washout phase after i.a. administration (Fig. 4). This indicates that receptor saturation might be a limiting factor for selective i.a. PRRT. It has been previously observed that tumor uptake of DOTATOC is influenced by the total amount of administered peptide which depends on receptor occupancy (de Jong et al. 1999, Müller et al. 2007). Such mass effects are often more relevant in receptor targeted drugs compared with metabolized drugs (Jagoda et al. 2004). Unfortunately, the risk of receptor saturation increases when the agent is delivered directly into the arterial supply of the tumor. Further investigation will be necessary to determine the maximum amount of peptide that can be given per treatment cycle. The observed washout effect from the target region also reduces the improvement in tumor uptake after i.a. administration over time. Nevertheless, in comparison to the i.v. route, we demonstrate higher tumor uptake $4 \mathrm{~h}$ and even $72 \mathrm{~h}$ after i.a. infusion despite the washout effect. Therefore, a relevant improvement in tumor dose was predictable for the therapy with ${ }^{90} \mathrm{Y}$ - or ${ }^{177} \mathrm{Lu}$-labeled DOTATOC. However, the relative improvement in tumor dose generated by i.a. administration could be more pronounced if radionuclides with a shorter half-life would be introduced for PRRT.

Within the limited number of patients of our pilot study radiologic $\mathrm{CR}$ and $\mathrm{PR}$ (decline of $>50 \%$ of lesions sum diameter) was observed in $60 \%$ of the patients and correlated with a decrease in tumor markers. In contrast, the response rates for the i.v. regime are typically reported as $25-30 \%$ (Kwekkeboom et al. 2008, Bodei et al. 2010). Therefore, our regime seems to double the response rate. However, in addition to RECIST criteria, Kwekkeboom introduced the term 'minor response' $(>25-<50 \%$ decline in diameter) with another $16 \%$ of minor responders. In comparison to that, the improvement of the i.a. approach is rather gradual, shifting minor responders to RECIST responders. A significantly higher response of pancreatic in comparison to small intestinal NETs was demonstrated for the i.v. PRRT (Pfeifer et al. 2011). According to the observation of Pfeifer et al. (2011), we also observed a remarkably higher response rate in pancreatic NET (patients no. 2-9), whereas liver metastases arising from the intestine (patients no. 11-13 and 15) were classified as stable. For our 
patients, median TTP was not reached with a mean follow-up period of 20 months. Therefore, our results compare favorably to, e.g. the NET treatment with Everolimus and Sunitinib (Raymond et al. 2011, Yao et al. 2011) that reported a median progression-free survival of 11-12 months, in pancreatic NETs respectively. However, to demonstrate a survival benefit in comparison to systemic PRRT, with a reported TTP of 40 months (Kwekkeboom et al. 2008), another 2 years of follow-up would be necessary.

A relationship between tumor dose and response rate has already been indicated for systemic radiopeptide therapy (Pauwels et al. 2005); therefore, the improvement observed with i.a. infusion is in accordance with the expected dose-response. However, in this study, only patients with liver dominant disease were included. This may have led to a selection bias toward patients with less tumor burden than in the studies involving i.v. PRRT. On the other hand, liver metastases are frequently the life-limiting problem for patients with metastatic NET. Also, patients with low tumor burden may especially benefit from neoadjuvant radiopeptide therapy since it may convert initially inoperable NETs to resectable ones (Stoeltzing et al. 2010). Thus, i.a. radiopeptide therapy offers substantially higher clinical improvement in patients with liver-confined NET metastases.

The observed side effects from i.a. radiopeptide therapy included moderate acute hematologic toxicity - typically grades I and II according to CTAE-criteria. This equals the toxicity profile of i.v. radiopeptide therapies. No acute kidney toxicity was observed. However, the decline of renal function with radiopeptide therapy is chronic (Bodei et al. 2008) and our long-term follow-up is still ongoing. Nevertheless, kidney radiation might be partly avoided with the i.a. approach. With i.a. therapy some amount the radiopeptide is partly trapped within the tumor during the first pass and does not reach the systemic circulation thus mitigating renal damage.

Even though the radiopeptide was administered directly into the hepatic artery, no acute liver toxicity was observed. Therefore, the side effects of i.a. PRRT might be actually lower than with other established locoregional therapies, such as TAE, TACE, or SIRT probably due to the specific, receptor-targeted character of the radiopeptide. Furthermore, the patients who were classified as responders presented with a relevant decline of $\gamma \mathrm{GT}$ and transaminases. Since liver metastases often are the life-limiting factor, these improvements in liver parameters are encouraging.

\section{Conclusion}

In this pilot study, the i.a. administration of PRRT resulted in a higher rate of objective radiologic responses than reported for the i.v. regime. However, this observation has still to be proven with a higher number of patients. Our data indicate that the maximum achievable tumor uptake might be limited by receptor saturation. Therefore, high specific activity is pivotal for this kind of therapy and further investigation will be necessary to improve the treatment regime. While only selected patients are eligible for i.a. administration of PRRT, this group of patients could greatly benefit from this approach.

\section{Declaration of interest}

The authors declare that there is no conflict of interest that could be perceived as prejudicing the impartiality of the research reported.

\section{Funding}

This research did not receive any specific grant from any funding agency in the public, commercial or not-for-profit sector.

\section{Acknowledgements}

We thank all the physicians and technicians of the Department of Nuclear Medicine and of the Department of Diagnostic and Interventional Radiology, the staff of the patient unit RAD4 and our colleges in the radiopharmacy who participated in patient care, diagnostic imaging, angiography, and assistance in conducting the therapies or preparation of the radiopharmaceuticals.

\section{References}

Bodei L, Cremonesi M, Ferrari M, Pacifici M, Grana CM, Bartolomei M, Baio SM, Sansovini M \& Paganelli G 2008 Long-term evaluation of renal toxicity after peptide receptor radionuclide therapy with ${ }^{90}$ Y-DOTATOC and ${ }^{177}$ Lu-DOTATATE: the role of associated risk factors. European Journal of Nuclear Medicine and Molecular Imaging 35 1847-1856. (doi:10.1007/ s00259-008-0778-1)

Bodei L, Pepe G \& Paganelli G 2010 Peptide receptor radionuclide therapy (PRRT) of neuroendocrine tumors with somatostatin analogues. European Review for Medical and Pharmacological Sciences 14 347-351.

Jagoda EM, Vaquero JJ, Seidel J, Green MV \& Eckelman WC 2004 Experiment assessment of mass effects in the rat: implications for small animal PET imaging. Nuclear Medicine and Biology 31 771-779. (doi:10.1016/j. nucmedbio.2004.04.003) 
de Jong M, Breeman WA, Bernard BF, van Gameren A, de Bruin E, Bakker WH, van der Pluijm ME, Visser TJ, Mäcke HR \& Krenning EP 1999 Tumour uptake of the radiolabelled somatostatin analogue (DOTA0, TYR3) octreotide is dependent on the peptide amount. European Journal of Nuclear Medicine 26 693-698. (doi:10.1007/ s002590050439)

de Jong M, Breeman WA, Valkema R, Bernard BF \& Krenning EP 2005 Combination radionuclide therapy using ${ }^{177} \mathrm{Lu}$ - and ${ }^{90} \mathrm{Y}$-labeled somatostatin analogs. Journal of Nuclear Medicine 46 13S-17S.

Kratochwil C, Giesel FL, López-Benítez R, Schimpfky N, Kunze K, Eisenhut M, Kauczor HU \& Haberkorn U 2010 Intraindividual comparison of selective arterial versus venous ${ }^{68} \mathrm{Ga}$-DOTATOC PET/CT in patients with gastroenteropancreatic neuroendocrine tumors. Clinical Cancer Research 16 2899-2905. (doi:10.1158/10780432.CCR-10-0004)

Kwekkeboom DJ, de Herder WW, Kam BL, van Eijck CH, van Essen M, Kooij PP, Feelders RA, van Aken MO \& Krenning EP 2008 Treatment with the radiolabeled somatostatin analog [ ${ }^{177}$ Lu-DOTA0, Tyr3] octreotate: toxicity, efficacy, and survival. Journal of Clinical Oncology 26 2124-2130. (doi:10.1200/JCO.2007.15. 2553)

Modlin IM, Latich I, Kidd M, Zikusoka M \& Eick G 2006 Therapeutic options for gastrointestinal carcinoids. Clinical Gastroenterology and Hepatology 4 526-547. (doi:10.1016/j.cgh.2005.12.008)

Müller C, Forrer F, Bernard BF, Melis M, Konijnenberg M, Krenning EP \& de Jong M 2007 Diagnostic versus therapeutic doses of ((177)Lu-DOTA-Tyr(3))-octreotate: uptake and dosimetry in somatostatin receptor-positive tumors and normal organs. Cancer Biotherapy \& Radiopharmaceuticals 22 151-159. (doi:10.1089/cbr.2007.309)

Pauwels S, Barone R, Walrand S, Borson-Chazot F, Valkema R, Kvols LK, Krenning EP \& Jamar F 2005 Practical dosimetry of peptide receptor radionuclide therapy with (90)Y-labeled somatostatin analogs. Journal of Nuclear Medicine 46 92S-98S.

Pfeifer AK, Gregersen T, Grønbæk H, Hansen CP, MüllerBrand J, Herskind Bruun K, Krogh K, Kjær A \& Knigge U 2011 Peptide receptor radionuclide therapy with Y-DOTATOC and (177)Lu-DOTATOC in advanced neuroendocrine tumors: results from a Danish cohort treated in Switzerland. Neuroendocrinology 93 189-196. (doi:10.1159/000324096)
Raymond E, Dahan L, Raoul JL, Bang YJ, Borbath I, Lombard-Bohas C, Valle J, Metrakos P, Smith D, Vinik A et al. 2011 Sunitinib malate for the treatment of pancreatic neuroendocrine tumors. New England Journal of Medicine 364 501-513. (doi:10.1056/NEJMoa1003825)

Rinke A, Müller HH, Schade-Brittinger C, Klose KJ, Barth P, Wied M, Mayer C, Aminossadati B, Pape UF, Bläker M et al. 2009 Placebo-controlled, double-blind, prospective, randomized study on the effect of octreotide LAR in the control of tumor growth in patients with metastatic neuroendocrine midgut tumors: a report from the PROMID Study Group. Journal of Clinical Oncology 27 4656-4663. (doi:10.1200/JCO.2009.22.8510)

Rolleman EJ, Valkema R, de Jong M, Kooij PP \& Krenning EP 2003 Safe and effective inhibition of renal uptake of radiolabelled octreotide by a combination of lysine and arginine. European Journal of Nuclear Medicine and Molecular Imaging 30 9-15. (doi:10.1007/s00259-0020982-3)

Stoeltzing O, Loss M, Huber E, Gross V, Eilles C, MuellerBrand J \& Schlitt HJ 2010 Staged surgery with neoadjuvant ${ }^{90}$ Y-DOTATOC therapy for down-sizing synchronous bilobular hepatic metastases from a neuroendocrine pancreatic tumor. Langenbeck's Archives of Surgery 395 185-192. (doi:10.1007/s00423-009-0520-x)

Strosberg JR, Fine RL, Choi J, Nasir A, Coppola D, Chen DT, Helm J \& Kvols L 2011 First-line chemotherapy with capecitabine and temozolomide in patients with metastatic pancreatic endocrine carcinomas. Cancer 117 268-275. (doi:10.1002/cncr.25425)

Velikyan I, Sundin A, Eriksson B, Lundqvist H, Sörensen J, Bergström M \& Långström B 2010 In vivo binding of $\left[{ }^{68} \mathrm{Ga}\right]$-DOTATOC to somatostatin receptors in neuroendocrine tumours-impact of peptide mass. Nuclear Medicine and Biology 37 265-275. (doi:10.1016/j. nucmedbio.2009.11.008)

Yao JC, Shah MH, Ito T, Bohas CL, Wolin EM, Van Cutsem E, Hobday TJ, Okusaka T, Capdevila J, de Vries EG et al. 2011 Everolimus for advanced pancreatic neuroendocrine tumors. New England Journal of Medicine 364 514-523. (doi:10.1056/NEJMoa1009290)

Received in final form 21 July 2011

Accepted 26 July 2011

Made available online as an Accepted Preprint 26 July 2011 